\title{
The Measure of Success: Constraints, Objectives, and Tradeoffs in Morphogen-mediated Patterning
}

\author{
Arthur D. Lander, Wing-Cheong Lo, Qing Nie, and Frederic Y.M. Wan \\ Departments of Developmental and Cell Biology, Mathematics and Biomedical Engineering, and \\ Center for Complex Biological Systems, University of California, Irvine, California 92697-2300 \\ Correspondence: adlander@uci.edu
}

\begin{abstract}
A large, diverse, and growing number of strategies have been proposed to explain how morphogen gradients achieve robustness and precision. We argue that, to be useful, the evaluation of such strategies must take into account the constraints imposed by competing objectives and performance tradeoffs. This point is illustrated through a mathematical and computational analysis of the strategy of self-enhanced morphogen clearance. The results suggest that the usefulness of this strategy comes less from its ability to increase robustness to morphogen source fluctuations per se, than from its ability to overcome specific kinds of noise, and to increase the fraction of a morphogen gradient within which robust threshold positions may be established. This work also provides new insights into the longstanding question of why morphogen gradients show a maximum range in vivo.
\end{abstract}

In recent years, much research on morphogen gradients has shifted from purely mechanistic questions-how gradients form and how morphogens signal — to strategic ones-how gradients perform well in the face of various kinds of constraints and perturbations. Forty years ago, Francis Crick was among the first to call attention to constraints that morphogens face, noting that the time required to spread a signal by random transport through a tissue varies with the square of distance (Crick 1970). Using order-of-magnitude calculations, he argued that observed biological maxima for morphogen-mediated patterning were just about where they should be if morphogen signals spread by aqueous diffusion.
Although the idea that diffusion time is what limits the sizes of morphogen gradients remains untested, Crick's work established a precedent of seeking explanations for developmental processes in terms of constraints imposed by the physical world. In the area of biological pattern formation, continued interest in how real-world limits constrain mechanisms has led many current investigators to focus on matters of robustness, the engineering term that describes the relative insensitivity of a system's behavior to perturbations it may be expected to encounter. With respect to morphogen gradients, most work has focused on parametric robustness, i.e., insensitivity to parameter values (e.g., the dosage of genes,

Editors: James Briscoe, Peter Lawrence, and Jean-Paul Vincent

Additional Perspectives on Generation and Interpretation of Morphogen Gradients available at www.cshperspectives.org

Copyright (C) 2009 Cold Spring Harbor Laboratory Press; all rights reserved; doi: 10.1101/cshperspect.a002022

Cite this article as Cold Spring Harb Perspect Biol 2009;1:a002022 
A.D. Lander et al.

levels, or rate constants of enzymes [Eldar et al. 2002; Eldar et al. 2003; Eldar et al. 2004; Bollenbach et al. 2005; Shimmi et al. 2005; White et al. 2007]). Some investigators have also focused on the "precision" of morphogen gradients, which may be understood as robustness to the causes and effects of natural variation among individuals in a population (Houchmandzadeh et al. 2002; Gregor et al. 2007; Tostevin et al. 2007; Bollenbach et al. 2008; Emberly 2008).

Remarkably, after hardly a decade of intensive study of such questions, we find ourselves awash in a sea of diverse and intriguing mechanisms for conferring one or another type of robustness on morphogen-mediated patterning. Mechanisms that operate at the level of gradient formation include self-enhanced morphogen degradation (Eldar et al. 2003), facilitated transport (Eldar et al. 2002; Shimmi et al. 2005), serial transcytosis (Bollenbach et al. 2005), presteady state patterning (Bergmann et al. 2007), and competition between morphogens for binding to inhibitors (Ben-Zvi et al. 2008). Mechanisms that operate at the level of morphogen detection and interpretation include morphogenetic apoptosis (Adachi-Yamada and O'Connor 2002), cell rearrangement (Ashe and Briscoe 2006), integration of signals from multiple morphogens (McHale et al. 2006; Morishita and Iwasa 2008), and various types of local cell-to-cell signaling (e.g., Amonlirdviman et al. 2005).

Why so many strategies? Biologists are often quick to ascribe multiplicity to redundancy, but the perspective of engineering suggests a different view. Most engineers accept the "no free lunch" principle (also referred to as "conservation of fragility"), which states that any mechanism that increases robustness in one setting (i.e., to one type of perturbation, or with respect to one type of output) always compromises it in another. The fact that every strategy comes at a price has been offered as an explanation for the seemingly inescapable fragility of highly engineered, modern technology (Carlson and Doyle 2002). By building complex machines that resist everything we think of, we inevitably create susceptibilities to the things we neglected. Although biology is not the result of human engineering, we have no reason to believe that natural selection can circumvent the limits that engineers confront.

In a world of no free lunch, one must evaluate a strategy not just by what it is good for, but the "price" of using it. With regard to morphogen-mediated patterning, it is reasonable to suggest that diverse strategies exist because each comes at a different price. If so, achieving meaningful biological understanding requires that we engage in a sort of cost-benefit analysis, in which each strategy is evaluated in the context of the performance objectives of the organism and constraints of the physical world. This is a tall order, as there is a great deal we still do not know about the performance needs of developing organisms (for example, for all the work performed so far on morphogen gradient robustness, we still know little about the magnitudes of the perturbations that need to be withstood). Nevertheless, there is no reason not to get started, as even through the early investigation of hard questions, one commonly learns useful things.

\section{SELF-ENHANCED CLEARANCE: A PARADIGM STRATEGY FOR ROBUSTNESS}

In this vein, we begin by reviewing a wellaccepted strategy for achieving parametric robustness in morphogen gradients: the principle of self-enhanced clearance (SEC). In their seminal paper, Eldar et al. (2003) described how having a morphogen stimulate its own degradation can provide a powerful way to build gradients that are highly robust to variation in the level of morphogen production at a source (although they referred explicitly to degradation, it is actually the removal of the morphogen from the diffusing pool-e.g., by binding and endocytosis, which may or may not be accompanied right away by degradation-that makes the strategy work, hence our substitution of the term "clearance" for degradation). These investigators found evidence for SEC in two of the well-known gradients in the Drosophila wing imaginal disc-those formed by Wingless ( Wg) and 
Hedgehog (Hh). In both cases, albeit by different molecular mechanisms, morphogen signaling leads to increased clearance, such that fluctuations in morphogen production are effectively neutralized close to the source. Recently, SEC has also been recognized in retinoic acid gradients in the zebrafish embryo (White et al. 2007).

Curiously, the third major gradient in the Drosophila wing disc - the bone morphogenetic protein (BMP) gradient (formed primarily by the morphogen Dpp, with assistance from the related TGF-beta family member Gbb [Khalsa et al. 1998]) — not only fails to engage in SEC, it does the opposite: BMP signaling downregulates expression of the BMP receptor thickveins $(\mathrm{Tkv})$, and it is this receptor that provides the major route for BMP clearance. The contrast between the relationship between signaling and clearance in the BMP versus $\mathrm{Wg}$ gradients of the wing disc is strikingly illustrated by the opposite results obtained when receptors are overexpressed in the two systems. Forced overexpression of $\mathrm{Wg}$ receptor gene $D f z 2$ leads to a marked expansion of the Wg signaling gradient, whereas forced overexpression of $T k v$ leads to a marked contraction of the BMP gradient (Cadigan et al. 1998; Lecuit and Cohen 1998).* More recently, it was also shown that reduced $T k v$ expression expands the BMP signaling gradient (Akiyama et al. 2008).

By the same token that SEC promotes robustness (to morphogen production rates), self-repressed clearance ought to do the opposite. We wondered why the BMP gradient would use a seemingly counter-productive strategy. This prompted a closer look at the details of gradient formation.

\footnotetext{
*The stabilizing effects of $D F z 2$ on the Wg gradient do not necessarily mean that DFz2 is not a clearance receptor for Wg. As Eldar et al. (2003) point out, SEC in the Wg gradient requires an "active" mechanism, wherein Wg signaling regulates some process that influences the efficiency of $\mathrm{Wg}$ removal. Although they suggested that DFz2 might sequester a protease, recent work suggests that regulation of accessory molecules such as notum or dally-like, that have marked effects on $\mathrm{Wg}$ gradient formation (Giraldez et al. 2002; Han et al. 2005), could be involved.
}

As a start, we recall that, in a tissue with uniform morphogen decay, with morphogen produced by a point source (in one dimension) or line source (in two dimensions), the gradient that is formed will take on, at steady state, the shape of a declining exponential function (equation 1, see Box 1). A single parameter $\lambda$ captures the characteristic length scale of such a system (distance over which the gradient decreases by $e^{-1} \sim 37 \%$ ); it relates the processes of diffusion and decay through the formula $\lambda=(D / k)^{2}$, in which $D$ is the effective diffusion coefficient, and $k$ an effective clearance rate constant. Rearranging equation 1 tells us that the location at which any threshold value of morphogen $L$ is crossed in a uniform decay (UD) gradient is $x=-\lambda \ln \left(L / L_{0}\right)$. As many have pointed out, this means an $m$-fold change in $L_{0}$ produces an absolute shift in $x$ of $\lambda \ln m$, for all $x$. Because most morphogen gradients seem to pattern fields on the order of three length-scales in size (Reeves et al. 2006), this means a twofold variation in morphogen synthesis will displace all patterns by about one fourth of the width of the entire patterned field. It is this calculation that suggests that UD gradients, in general, lack the robustness that biology demands.

If, on the other hand, one makes decay nonuniform, clearing morphogen faster when morphogen levels are higher, the result is a nonuniform length scale-short close to the source and longer far away (equation 2). Although the pattern shift caused by a change in morphogen level at the source is still proportional to length scale, it is only the length scale near the source that matters. Because length scale at that location can be made very short, whereas length scales elsewhere are long, SEC gradients are more robust than UD gradients of similar size (Eldar et al. 2003).

Although this explanation captures the gist of SEC, it glosses over some important issues. In principle, there is no reason why a UD gradient could not use just as short a length scale as an SEC gradient. Suppose one wanted to position a patterning threshold $20 \mu \mathrm{m}$ from a morphogen source. In a UD gradient with $\lambda=10 \mu \mathrm{m}$, that threshold occurs at $2 \lambda$, 
A.D. Lander et al.

\section{BOX 1. MODELING THE SHAPES OF MORPHOGEN GRADIENTS}

The general equation for a morphogen gradient is $\partial_{t} c=D \partial_{x}^{2} c-F(c)+V(x)$, in which $c$ is morphogen concentration, $x$ is distance, $D$ is a diffusion coefficient, $V(x)$ is a rate of morphogen production (taken to be 0 for $x>0$ and $V$ for $-d<x<0$, in which $d$ is the width of the production region), and $F$ is a function that relates morphogen concentration to morphogen clearance. If receptors mediate clearance, do so linearly, and are not saturated, $F(c)=k c r / K_{m}$, in which $k$ is a rate constant, $r$ is receptor concentration, and $K_{m}$ is a modified dissociation constant. Defining a scaled variable $y=c / K_{m}$, we obtain the steady-state equation $y^{\prime \prime}=\frac{1}{\bar{\lambda}^{2}}(y-v[x])$, in which $\bar{\lambda}=\sqrt{D K_{m} /(k r)}$, and $v[x]=V(x) /(k r)$. This may be solved to

$$
y=y 0 e^{-x / \bar{\lambda}}
$$

If there is self-enhanced clearance (Eldar et al. 2003), such that decay increases with some power $n$ of $c$, then $F(c)=k c^{n+1}$. Making assumptions and substitutions as before, the steady state equation is $y^{\prime \prime}=\frac{1}{\bar{\lambda}^{2}}\left(y^{n+1}-v[x]\right)$, in which $\bar{\lambda}=\sqrt{D K_{m} /\left(k r^{n+1}\right)}$, and $v[x]=V(x) /\left(k r^{n+1}\right)$. For $n=1$, the solution is:

$$
y=\left(\frac{x}{\sqrt{6} \bar{\lambda}}+\frac{1}{\sqrt{y 0}}\right)^{-2}
$$

From these equations, we may obtain expressions for length scale $\lambda$ (as a function of $y$ ), the sensitivity $S_{x, y 0}$, of any position $x$ to the value of $y$ at $x=0$, and the transition width, $w$, caused by receptor binding noise. For equation $1, \lambda=\bar{\lambda}, S_{x, y 0}=\bar{\lambda} / x$, and $w=2 \bar{\lambda} / \sqrt{\rho y}$, in which $\rho$ is receptor concentration in numbers of molecules per cell. For equation $2, \lambda=\bar{\lambda} \sqrt{3 /(2 y)}, S_{x, y 0}=\frac{\bar{\lambda}}{x} \sqrt{\frac{3}{2 y 0}}$, and $w=\frac{\bar{\lambda}}{y} \sqrt{\frac{6}{\rho}}$.

The above solutions apply only under conditions of low receptor saturation, when levels of bound morphogen are proportional to those of free morphogen. More general forms for UD and SEC gradients are shown below, together with an example of self-repressed clearance (SRC). Although none of these can be solved explicitly, expressions for $\lambda, S_{x, y 0}$, and $w$ may still be obtained.

\begin{tabular}{lll}
\hline Case & Transformed equation & $\lambda(y)$ \\
\hline UD & $y^{\prime \prime}=\frac{1}{\bar{\lambda}^{2}}\left(\frac{y}{1+y}-v[x]\right)$ & $\frac{y \bar{\lambda}}{\sqrt{2} \sqrt{y-\ln (1+y)}}$ \\
SEC1 & $y^{\prime \prime}=\frac{1}{\bar{\lambda}^{2}}\left(\frac{y^{2}}{(1+y)^{2}}-v[x]\right)$ & $\frac{y \bar{\lambda}}{\sqrt{2} \sqrt{y+\frac{y}{1+y}-2 \ln (1+y)}}$ \\
SEC2 & $y^{\prime \prime}=\frac{1}{\bar{\lambda}^{2}}\left(\frac{y-\beta}{(1+y)}-v[x]\right)$ & $\frac{y \bar{\lambda}}{\sqrt{2} \sqrt{y-(1+\beta) \ln (1+y)}}$ \\
SEC3 & $y^{\prime \prime}=\frac{1}{\bar{\lambda}^{2}}\left(\frac{y^{2}}{(1+y)}-v[x]\right)$ & $\frac{y \bar{\lambda}}{\sqrt{(-2+y) y+2 \ln (1+y)}}$ \\
SRC1 & $y^{\prime \prime}=\frac{1}{\bar{\lambda}^{2}}\left(\frac{2 y}{1+y} /\left(1+\frac{\sqrt{1+\varphi^{2} y}}{\sqrt{1+y}}\right)-v[x]\right)$ & $\frac{y \bar{\lambda}}{2} / \sqrt{\ln \left[\frac{-1+\sqrt{(1+y)\left(1+y \varphi^{2}\right)}}{1+\varphi^{2}+2 \varphi\left(y \varphi+\sqrt{(1+y)\left(1+y \varphi^{2}\right)}\right.}\right]}$ \\
\end{tabular}

SEC1, the most directly comparable to equation 2, comes from replacing $F(c)=k c^{2}$ with $F(c)=k b^{2}$, in which $b$, the concentration of bound morphogen receptor complexes $=c r /\left(K_{m}+c\right)$. We note, however, that the most plausible mechanisms by which morphogen signaling might up-regulate 
morphogen removal are not consistent with this scenario. That is because, in most cases, the ratelimiting step in morphogen decay is morphogen capture, and if morphogen signaling stimulates that process, i.e., increases the association rate constant $k_{\text {on }}$ for morphogen-receptor interaction, then $K_{m}$ is no longer a constant, but a function of $b$. If we model this by replacing $k_{\text {on }}$ with $k_{\text {on }} /$ $(1+\alpha / b)$, we obtain SEC2, in which $\beta$ stands for $\alpha / r$. An alternative mechanism might be to allow morphogen signaling to up-regulate the expression of an enzyme that degrades free morphogen: In this case, receptor saturation would limit morphogen signaling, but not morphogen decay, leading to the expression $F(c)=k c b$, and equation SEC3, in which $\bar{\lambda}=\sqrt{D /(k r)}$. A third mechanismhaving morphogen signaling up-regulate receptor expression, as occurs in Hedgehog gradientsproduces a highly distinctively shaped, short-range gradient profile, and has not been considered here. The equation for self-repressed clearance, SRC1, was obtained by allowing morphogen signaling to exert negative feedback on receptor synthesis according to $r=r_{\max } /(\mathrm{I}+\gamma b)$, in which $r_{\max }$ is the total receptor level in the absence of feedback, $\bar{\lambda}=\sqrt{D K_{m} /\left(k r_{\max }\right)}$ and $\varphi=\sqrt{1+4 \gamma r_{\max }}$.

meaning that a twofold change in morphogen concentration at the source produces a $6.9 \mu \mathrm{m}$ $(=10 \ln 2)$ shift in pattern, or about $35 \%$ of the original distance to the threshold-hardly what one would call robust. But if the gradient used a length scale $\lambda=2 \mu \mathrm{m}$, so that the threshold occurred at $10 \lambda$, then a twofold change in morphogen concentration would produce only a $1.4 \mu \mathrm{m}$ shift (6.9\%).

Notice that we have chosen to quantify robustness in terms of relative (percentage) movement, not absolute movement, of a patterning threshold location. By this metric, no single value characterizes an entire gradient; robustness is different at different patterning thresholds. We find this approach quite natural-after all, a $2 \mu \mathrm{m}$ change in a $4 \mu \mathrm{m}$ wide pattern element seems like a much more serious defect than a $2 \mu \mathrm{m}$ change in an $80 \mu \mathrm{m}$ wide pattern element. Moreover, it allows us to formalize the notion of robustness in terms of the standard engineering notion of sensitivity coefficient (Reeves and Fraser 2009), the measure of the fold-change in system output with respect to any fold-change in input (specifically, $S_{a, b}=d \ln a / d \ln b$ ). Sensitivity coefficients are unitless, facilitating comparisons among models and mechanisms. By this measure, in any morphogen gradient in which morphogen production is restricted to a domain $x<0$, the sensitivity of any threshold position $x>0$ to the level of morphogen at $x=0$ is simply $\lambda_{0} / x$, in which $\lambda_{0}$ stands for the length scale at the source.
Because a UD gradient can match, point for point, the robustness of an SEC gradient (simply by matching the length scale at the source), what is the advantage of nonuniform decay? As Figure 1A shows, such a UD gradient decreases, at every location, to a lower fraction of its starting value than the SEC one. The further from the source, the lower that fraction. For example, at the location where the sensitivity coefficient $=0.3$ (i.e., a twofold change in morphogen level at $x=0$ causes a $2^{0.3}$-fold [23\%] positional shift), morphogen levels have declined four times as much in the UD case as in the SEC one (compare arrows in Fig. 1A).

Presumably, there must be some limit to how low morphogen concentrations may get before they can no longer be useful. Accordingly, having to work with lower morphogen concentrations would seem a liability for UD gradients. But could not this liability be neutralized simply by using higher morphogen levels at the outset (i.e., near the source)? As shown in Figure 1B, any UD gradient described by equation 1 can always be made to match the robustness and morphogen level of any SEC gradient described by equation 2 , at any single location, simply by adjusting the initial value from which the UD gradient declines. Why then should a morphogen gradient bother with SEC?

\section{HOW HIGH IS TOO HIGH?}

The obvious answer is that there may be limitations to how high one can go in the level of 
A.D. Lander et al.
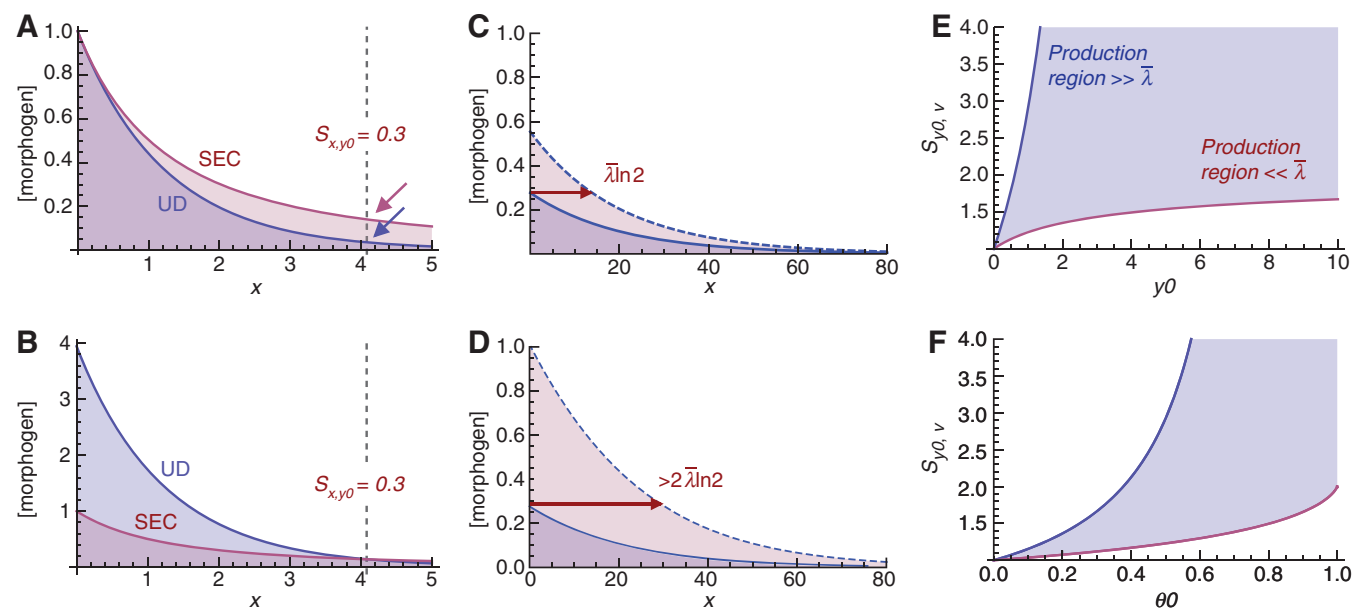

Figure 1. Gradient decay mechanisms and robustness to rates of morphogen synthesis. $(A)$ According to equations 1 and 2, morphogen gradients with uniform decay (UD) and self-enhanced clearance (SEC) will be equally robust to morphogen synthesis rates if they display the same length scale near the source. If they start from the same initial concentration, however, the UD gradient will fall increasingly below the SEC gradient as one moves further from the source (arrows show morphogen levels at the location where $S_{x, y 0}$, the sensitivity of threshold location to initial morphogen concentration, equals 0.3 ). (B) For any UD gradient, equivalent robustness to morphogen synthesis rate and equivalent morphogen levels at any single threshold point can be achieved by initiating the UD gradient from a higher starting value. $(C)$ According to equations 1 and 2, the amount by which a UD or SEC gradient shifts in response to a twofold change in morphogen synthesis is $\ln 2$ times the length scale at the source. However, outside the regime of very low receptor saturation, the shift can be much larger, as shown in $(D)$. (D) Units of free morphogen concentration are scaled to $K_{\mathrm{m}}$, such that the value of 0.28 corresponds to $22 \%$ receptor saturation. $(E, F)$ When receptor saturation is not negligible, morphogen concentration near the source can go up steeply with morphogen synthesis rate. $S_{y 0, v}$ is the coefficient of sensitivity of $y 0$ (morphogen level at $x=0$, scaled to $K_{\mathrm{m}}$ ) to the rate of morphogen synthesis, and is plotted in panel $E$ as a function of $y_{0}$ and in panel $F$ as a function of $\theta 0$, receptor saturation at $x=0$. Note that the results depend on the size of the morphogen production region relative to the length scale parameter, which determines the fraction of morphogen molecules that are cleared within the production region, as opposed to diffusing away from it.

morphogen at the start of a gradient. Certainly, proteins can only be synthesized and secreted so fast. However, given that morphogen gradients may be fed by morphogen-producing regions of considerable size (consult the Hh gradient in the Drosophila wing disc [Tabata and Kornberg 1994]), these limits are not especially constraining. To see what the real problem is, we need to strip away two assumptions that are widely used to simplify the analysis of morphogen gradients. These assumptions lay behind the formulation of equations 1 and 2 , as well as quite a few conclusions in the literature about how morphogen gradients behave.

The first assumption is that the level of morphogen receptor occupancy (the actual input to morphogen signaling) is, to a close approximation, proportional to that of free morphogen. This is only true when morphogen receptors are far from saturation. As levels of free morphogen increase, receptors become saturated, their fractional occupancy $\theta$ following the formula $\theta=c /\left(c+K_{\mathrm{m}}\right)$, in which $c$ is the concentration of free morphogen. The constant $K_{\mathrm{m}}$ here resembles the familiar dissociation constant for binding, but reflects modifications to account for effects of internalization and degradation on occupancy (Lander 1999). If receptors play any role in morphogen clearance (either directly or as a consequence of their signaling), then only when $\theta \ll 1$ everywhere are equations 1 and 2 valid. For larger $\theta$, gradient shape becomes distorted in a manner that, in principle, might be detectable 
in measurements of actual morphogen gradients. In practice, $\theta$ needs to be very close to 1 before such distortion is obvious. A more sensitive indication of receptor saturation is discrepancy between the measured length scale of a morphogen gradient and the measured length scale of its signaling gradient. A signaling gradient that declines more slowly than the gradient of the morphogen that elicits it would indicate possible receptor saturation near the source. Interestingly, when just such measurements were made for the Dpp gradient of the Drosophila wing disc (Bollenbach et al. 2008), length scales of $17 \pm 4.3 \mu \mathrm{m}$ for Dpp molecules, and $25 \pm 4.5 \mu \mathrm{m}$ for phosphorylated Mad (the signal arising from receptor occupancy), were obtained. Given the overlapping error bars, these measurements were reported as "not significantly different." Yet, given the distances over which the measurements were made, it is easy to show that, for a UD gradient, the observed 50\% increase in apparent length scale is just what one would observe if receptor occupancy is at about $50 \%$ near the source! Clearly, the possibility that real morphogen gradients start from a position of significant receptor saturation cannot be ignored.

The second assumption is that one may use the level of morphogen near the source as a valid proxy for the rate of morphogen production within the source. In studies of morphogen gradients, it is widely assumed that if one wishes to learn how robust a gradient will be to changes in morphogen production one need only calculate how robust it is to changes in its starting value (or, equivalently, the value of morphogen flux, or current, adjacent to the source). Strictly speaking, this assumption also only holds when receptors are far from saturation. For $\theta>0.25$, the deviation from ideal behavior is considerable, and it can be frankly massive if the width of the morphogen production region is large.

Figure 1C,D illustrates the point. Panel C uses equation 1 to plot the profile of free morphogen for a UD gradient with length scale $\lambda=20$. The dashed curve shows the effect of doubling the initial morphogen concentration, a rightward shift of $\lambda \ln 2$. Panel $D$, in contrast, plots a UD profile in which receptor saturation effects are taken into account. In this example, free morphogen concentration, $y$, is expressed relative to $K_{\mathrm{m}}$, so that the value of $\theta$ at $x=0$ equals $y 0 /(1+y 0)$, in which $y 0$ stands for the level of free morphogen at $x=0$. For example, because the solid curve in panel $\mathrm{D}$ starts at $y=$ 0.28 , receptor saturation at that point comes out to be $0.28 / 1.28=22 \%$. We also take the width of the morphogen production region into account, and assume it is large compared with the length scale of the morphogen within it. Under these circumstances, we find that a twofold increase in morphogen synthesis rate leads to a nearly fourfold increase in morphogen concentration next to the source, and more than a doubling of the rightward shift of the gradient.

Much of the explanation for this effect stems from a simple fact: If receptors mediate morphogen clearance, then as receptors become saturated, so does clearance. Accordingly, as morphogen synthesis increases, the fraction of synthesized molecules cleared by receptors goes down, meaning that the fraction of morphogen molecules that escape clearance within the production region (or nearby to it) goes up by more than the increase in synthesis.

We can quantify this effect with a sensitivity coefficient, $S_{y 0, v}$, which captures the sensitivity of $y 0$ to the rate of morphogen synthesis, $v$, in the production region. A value of $S_{y 0, v}=1$ corresponds to the statement that the level of free morphogen at $x=0$ varies linearly with the rate of morphogen synthesis; a higher value means a steeper-than-linear relationship. As shown in Figure 1E, $S_{y 0, v}$ is always greater than one for a UD gradient, and increases with increasing $y 0$. In Figure 1F, this relationship is plotted as a function of receptor saturation at $x=0$. From it, one can see that, with saturation levels just under $60 \%, S_{y 0, v}=4$, i.e., free morphogen can vary with as much as the fourth power of morphogen synthesis rate! For SEC gradients, the overall picture is similar for large $y_{0}$, although for values of $y_{0}<1, S_{y 0, v}$ dips below one (data not shown). This is because increasing receptor occupancy stimulates morphogen clearance (but only up to the point that receptors become saturated). 
A.D. Lander et al.

The advantage of quantifying the effects of receptor saturation and morphogen decay within production regions in terms of a sensitivity coefficient is that the quantity we are really after-the overall sensitivity of position $(x)$ to morphogen synthesis rate $(v)$ — can be found simply by multiplying $S_{y 0, v}$ by $S_{x, y 0}$ (the latter term is simply the sensitivity of position to $y 0$-the free morphogen concentration at $x=0$ - and equals $\lambda_{0} / x$, as discussed earlier). When we do this for UD gradients, we find that matching both the robustness and morphogen level of an SEC gradient is usually quite impossible. As one tries to raise $y 0$ to compensate for a small $\lambda$, the ensuing decline in robustness requires one to lower $\lambda$ even more.

What this means is that UD gradients cannot easily escape from the low morphogen levels they incur in exchange for robustness. But do they really need to? Until now, we have assumed that there is some fixed level of morphogen below which pattern formation cannot occur. In reality, this value is not fixed, but different for different types of gradient mechanism-e.g., UD versus SEC. To see why, we need to consider the effects of noise on morphogen gradient interpretation.

\section{HOW LOW IS TOO LOW?}

To the extent that morphogens specify tissue boundaries-locations to either side of which cells adopt different fates-the consequences of low morphogen levels on patterning need to be measured in terms of how they affect the ability to create such boundaries. We may distinguish two types of noise that influence the measurement of morphogen levels by cells: background noise and detection noise. The former refers to the degree of activation of the morphogen signaling pathway (or its downstream effectors) that occurs even in the absence of morphogen. The latter refers to inaccuracy in the measurement of morphogen levels because of noise in the measurement process itself. It is fair to say that we know very little about the levels of background noise among cells that respond to morphogens, but we may speculate that at least some of it comes from ligand-independent receptor signaling, which tends to increase with increasing receptor levels (Feng and Derynck 1996; Harris et al. 1999). Thus, background noise likely imposes constraints on the total numbers of receptors a cell may possess.

With regard to detection noise, we can distinguish at least three types: The first is stochastic variation in free morphogen concentration, because of morphogen molecules moving in and out of the volume around cells. The second is cell-to-cell variability in the machinery of detection (e.g., numbers of receptors per cell, rates of internalization, levels of signaling intermediates, cell size, etc.). The third is fluctuation in levels of receptor occupancy because of the stochastic nature of association, dissociation, and internalization. Because of the rapidity with which free molecules diffuse over short distances, it is likely that fluctuations in free morphogen concentration are too fast to have a significant impact on morphogen binding and signaling (i.e., the fluctuations average out over time [for discussion, see Lauffenburger and Linderman 1993]). Cell-to-cell variability could have much larger effects, depending on what is varying and on what time scales (see Bollenbach et al. 2008). In the case of fluctuations in receptor occupancy, because of the stochasticity of binding events, we can be much more precise, because we can model those events explicitly. As shown in Figure 2A, using rate constants in the range of those in known morphogen gradient systems, one predicts fluctuations that vary over many minutes to hours. Such slowly varying noise could have a pronounced impact on target gene expression, provided the magnitude is sufficient. As Figure 2A points out, the magnitude of such "binding noise" increases as receptor occupancy decreases - for Poisson processes the coefficient of variation ( $c v$; the standard deviation divided by mean), which is equal to the reciprocal of the square root of the mean.

The impact of detection noise on patterning (whether because of cell-to-cell variability or the stochastics of binding) is that some cells will find themselves on one side of a patterning threshold position, yet respond in the manner 


\section{A}
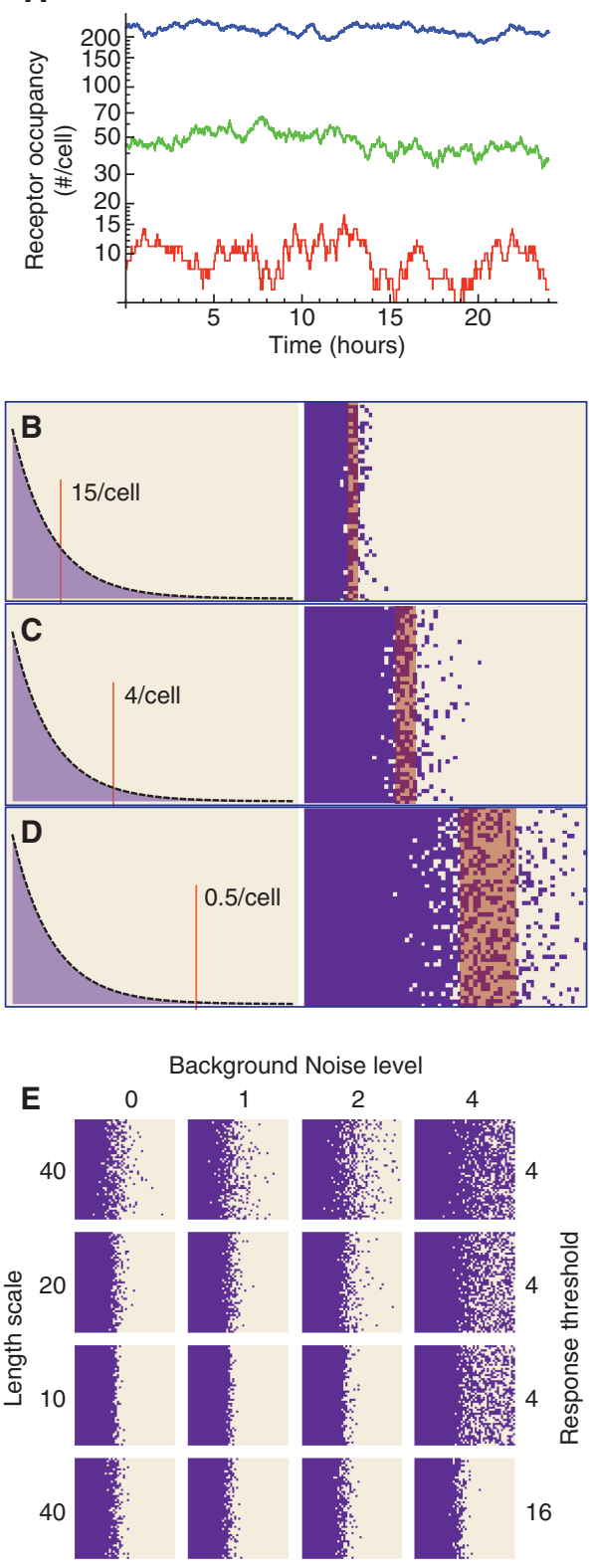

appropriate for cells on the other side. The result is a "salt-and-pepper" transition zone, rather than a sharp border of cell response, with a width equal to $2 c v \times \lambda$, in which $\lambda$ is the length scale of the gradient (Tostevin et al. 2007; Bollenbach et al. 2008; Emberly 2008) (for gradients in which length scale is not constant, $w \approx 2 c v \times \lambda^{*}$ in which $\lambda^{*}$ is the average length scale within the region). This makes
Figure 2. Binding noise and background noise in morphogen detection and patterning. (A) Stochastic simulations were performed to visualize predicted fluctuations in receptor occupancy on cells with mean occupancies of 9.1 (red), 45.5 (green), or 227 (blue) receptors per cell. A logarithmic axis is used to show that the relative contribution of binding noise goes down as occupancy goes up. For the rate parameters used here $\left(k_{\mathrm{deg}}=2 \times 10^{-4} / \mathrm{sec}\right.$ for bound receptors and $10^{-4} / \mathrm{sec}$ for free; $k_{\text {off }} \ll k_{\text {deg }}$; receptor synthesis rates of 72 [red], 360 [green], and 1800 [blue] molecules/cell/h), the time course of the fluctuations is on the order of an hour, and therefore likely to be physiologically relevant. $(B-D)$ Effect of binding noise on patterning. Simulations were performed to show the expected behavior of a field of $50 \times 70$ cells, exposed to an exponentially declining morphogen gradient with length scale of 10 cell diameters, in which initial morphogen concentration is sufficient to occupy 50 receptors per cell, and thresholds for activating gene expression (represented by a color change from light to dark) occur at occupancy levels of $15(B), 4(C)$, or $0.5(D)$ receptors per cell. As expected, the width of the variegated response region increases with lower occupancy thresholds; this is quantified by overlaid pink boxes, which mark the regions within which cells have more than a $15 \%$ chance of responding inappropriately for their position. (E) Effects of background noise. Simulations were performed as in $B-D$, except that background (Gaussian) noise was added at a mean level equivalent to the occupancy of 1,2 , or 4 receptors per cell, with a coefficient of variation of $30 \%$. The effects of background noise become significant only when it nears the cell response threshold. Whereas decreasing the gradient length scale is an effective strategy for minimizing the effects of binding noise, it has little effect on background noise, which can only be overcome by raising the response threshold.

intuitive sense: Even if there is a great deal of detection noise at a particular location, if the gradient is very steep, cells nearby will tend to have values of receptor occupancy well above or below the level of the noise. Figure 2B-D simulates the effects of binding noise on a UD gradient, in which thresholds for gene expression are set at levels of receptor occupancy of 15,4 , and 0.5 per cell. The pink regions mark 
A.D. Lander et al.

the boundaries outside of which cells have no more than a $15 \%$ chance of choosing their fates incorrectly.

The fact that many gene-expression boundaries are variegated (e.g., Moser and Campbell 2005; Bollenbach et al. 2008) supports the view that noise has a significant impact in vivo. Whether the dominant source is cell-to-cell variation or binding noise is likely to depend on location, as the magnitude of the former changes little with distance, whereas the latter grows dramatically as one moves away from a morphogen source (because receptor occupancy decreases). Thus, binding noise has the potential to limit on the distances over which morphogen gradients may be used, limits we will later argue are fairly easily reached.

Regardless of the source of detection noise, it should be apparent that SEC gradients should, in general, be more sensitive to it than UD gradients. This is because the length scales of SEC gradients increase with distance, whereas those of UD gradients do not. The very thing that makes SEC gradients more robust to one perturbation-fluctuations in the level of morphogen production-makes them more fragile to another-fluctuations in receptor occupancy. Indeed, if one compares SEC and UD gradients with the same length scales at the morphogen source, and asks how robust to morphogen synthesis rate they are at the most distant location where the transition width (because of binding noise) remains below any arbitrary value, one finds that SEC gradients perform only marginally better (Fig. 3A).

\section{IT IS NOT HOW ROBUST YOU MAKE IT, IT IS HOW YOU MAKE IT ROBUST}

In the space of a few pages, we have come from explaining how SEC is a powerful robustness strategy to arguing that it is marginally useful. The fact that both statements can be supported by evidence nicely makes our main point: The evaluation of performance is always a matter of context. A strategy cannot be judged by how well it does a single job, but by how it manages the tradeoffs that come with competing performance objectives (and the absence of free lunch). What we learned in this case is that, within a context established by two particular, similarly weighted objectives (robustness to morphogen synthesis rate, and binding noise), SEC seems not much better than UD. But perhaps this was not the right context within which to make the comparison?

The value of being able to reduce strategies to mathematical formulations is that it enables us to explore performance over a diverse range of contexts, homing in on those sets of situations in which a given strategy outperforms others. In the case of SEC, two such situations are easily found.

The first is when background noise is more significant than binding noise, at the locations where patterning thresholds are crossed. The reason for this is that background noise does not show the same relationship to length scale as detection noise. The corrupting influence of background noise increases extremely steeply as the signal-to-noise ratio approaches one; in this neighborhood, no amount of length-scale shortening can recover the signal. Only elevating the signal above the noise does the trick (Fig. 2E). This, of course is precisely what the SEC strategy does well: Point for point, for the same degree of robustness to morphogen synthesis rate, the SEC gradient will always have a higher level of morphogen, and thus rise higher above the background. At present, we know little about the levels of background or binding noise in morphogen gradients, so the data are not available to determine whether those gradients with high background noise are indeed ones that use SEC.

The second situation in which SEC really shines is when a morphogen needs to place patterning thresholds at widely separated positions. As Figure 3B points out, because sensitivity to morphogen production rate decreases with distance from the source, whereas detection noise (especially binding noise) increases with distance, most gradients should display a limited "useful patterning range" (the region between $x_{S}$ and $x_{w}$ in Fig. 3B). Between the source and this region, robustness is not sufficient, and beyond this region, noise is too high. In Figure 3C we calculate, for UD gradients, 
A

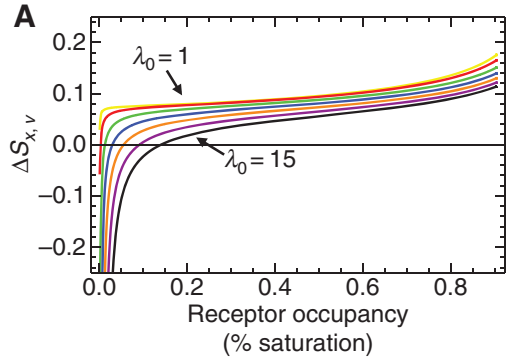

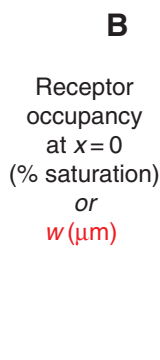

B

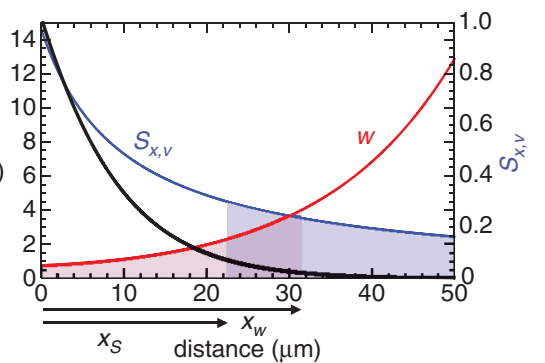

C

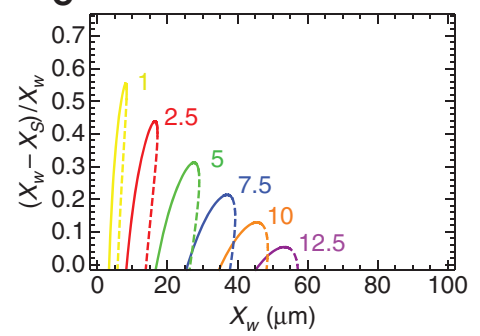

D

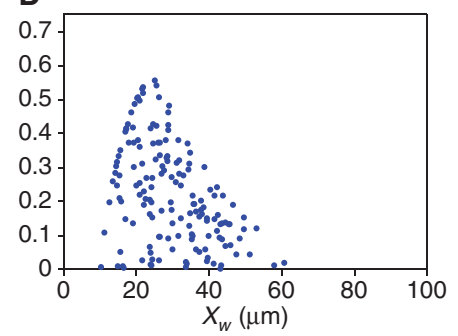

E

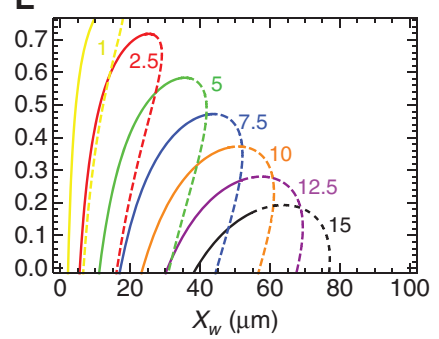

Figure 3. Comparing performance of different gradient strategies. (A) Exact expressions for $S_{x, v}$ (robustness of position with respect to morphogen synthesis rate) for UD and SEC gradient mechanisms were evaluated for a series of values of $\lambda_{0}$ (length scale at $x=0$ ), at the farthest location where the transition width $w$ (positional uncertainty because of binding noise), remains below a maximum allowable value (in this example, $4 \mu \mathrm{m}$, assuming a receptor density of $4500 /$ cell). $S_{x, v}$ for SEC1 gradients was subtracted from $S_{x, v}$ for UD gradients to yield $\Delta S_{x, v}$, the robustness improvement because of SEC. Under most circumstances, $\Delta S_{x, v}$ is relatively small $\left(\Delta S_{x, v}=0.1\right.$ means a twofold change in $v$ produces a $2^{0.1}$-fold $(7.2 \%)$ smaller change in $x$ in SEC vs. UD gradients). Values of $\lambda_{0}$, in micrometers, are 1 (yellow), 2.5 (red), 5 (green), 7.5 (blue), 10 (orange), 12.5 (purple), and 15 (black). For these calculations, the size of the morphogen production region was assumed to be $\ll \bar{\lambda}$ (which minimizes degradation of robustness) (Figure 1E,F). For different receptor levels, identical curves are obtained if $w$ is adjusted in proportion to the square root of the receptor level. (B) Receptor occupancy, robustness $\left(S_{x, v}\right)$, and transition width because of binding noise $(w)$, calculated empirically, for a simulated UD gradient ( $20 \mu \mathrm{m}$ production region; 4485 receptors per cell; $D=10 \mu \mathrm{m}^{2} / \mathrm{sec}$ ). Thresholds $x_{S}$, in which $S_{x, v}=0.3$, and $x_{w}$ in which $w=4$, are shown. Note that only a small fraction of the gradient lies between $x_{w}$ and $x_{S}$ (the "useful region"). (C) Predicted sizes of useful regions of UD gradients of different $\lambda_{0}$ (values shown beside each curve, color coded as in panel $A$ ), as a function of $x_{w}$ the maximum patterning width (calculations assume small production region). Moving clockwise around each loop, one encounters gradients with initial receptor occupancies, $\theta 0$, running from low to high (curves change from solid to dashed at the point where $\theta 0=0.5)$. $(D)$ A set of random UD gradient profiles (covering a wide range of parameter values) was generated as in panel $B$, and values of $x_{S}$ and $x_{w}$ were calculated empirically. Note the good agreement with panel $C$, except for very small $\lambda_{0}$ (when the assumption of small production region size is least valid). (E) Predicted sizes of useful regions of SEC1 gradients of different $\lambda_{0}$, plotted as in $C$. Note that in both $C$ and $E$, the size of the useful region is generally maximized when receptors are moderately saturated at the source $(\theta 0$ close to 0.5$)$. This provides a strong theoretical argument why real morphogen gradients are unlikely to operate under conditions in which the effects of receptor saturation can be neglected!

the relative proportion of the total patterning range that is "useful" in this way, as a function of gradient size and $\lambda_{0}$ (length scale near the source). The calculations imply that, to spread a gradient over a substantial distance, one must sacrifice a great deal of the useful patterning range. Figure 3D confirms the analysis in
Figure 3C with numerical simulations based on randomly chosen parameter sets.

Figure $3 \mathrm{E}$ goes through the same analysis as Figure 3C, but for SEC gradients (strategy SEC1) (see Box 1). The most striking difference is the much greater relative size of the "useful range." After seeing this result, it occurred to 
A.D. Lander et al.

us that one of the most significant differences between the wing disc Wg gradient (which uses SEC) and the wing disc Dpp gradient (which does not) is the fraction of the morphogen field in which patterning thresholds are crossed: Dpp creates gene expression boundaries at essentially two positions - with the closest (the sal boundary) being about $60 \%$ of the way to the farthest (the omb boundary). Thus, the useful range need only be about $40 \%$ of the distance to the omb boundary (Nellen et al. 1996; Moser and Campbell 2005). In contrast, Wg creates boundaries both very close to the morphogen source (e.g., sens) and very far away (e.g., Dll and $v g$ ), implying a very large useful range (Zecca et al. 1996; Neumann and Cohen 1997). This requirement alone may make the SEC strategy a much better choice than UD for the Wg gradient. It should be noted that the superior performance of SEC in this context is not divorced from its effects on parametric robustness (those effects play into where the boundaries of the useful patterning region end up). The point is, in this context, it is less the amount of robustness, than the way in which robustness is contributed, that matters.

\section{CONCLUDING REMARKS: HOW FAR IS TOO FAR?}

At the start of this article, we recalled Crick's conjecture that the relationship between diffusion time and distance might limit the range over which gradients pattern. From the data in Figure $3 \mathrm{C}-\mathrm{E}$, the reader may begin to see another reason why the maximum ranges of morphogen gradients should be constrained: As gradients get longer, the relative sizes of their useful regions decline, until eventually they vanish altogether. This occurs because at some point, the location where the noise is too great starts to occur before the location where robustness becomes adequate.

In Figure 3C, this occurs at about $62 \mu \mathrm{m}$ from the source, but the precise limiting value depends on two parameters that were chosen for the purpose of illustration. We could double this limit by quadrupling the allowable number of receptors per cell (to 18,000) or doubling the tolerable transition width (to $8 \mu \mathrm{m})$. Remarkably, changing the diffusion coefficient makes no difference (the maximum patterning range is essentially defined in terms of the units of the transition width). The point is that, for reasonable parameter choices, a patterning range of a few hundred microns - what is commonly observed in morphogen gradients-may well be the theoretical maximum.

Of course, this is not a "hard" limit in the sense of the one that Crick discussed. One can easily imagine strategies to circumvent it, even in UD gradients: With larger cells, more receptors per cell could probably be accommodated without producing too much background noise. By using molecules other than receptors to clear morphogens, higher levels of receptor saturation could be allowed without compromising robustness. Similar effects could be obtained by regulating clearance differently in morphogen-producing versus morphogenresponding regions. Does this explain why wing disc cells are so large for their diameters (i.e., long and thin)? Why expression of the proteoglycan dally within the Dpp production region of wing discs appears to decrease the amount of Dpp that escapes from that region (Fujise et al. 2003)? Why expression of Tkv receptors is markedly repressed in the same Dpp production region (Tanimoto et al. 2000)? Perhaps, but perhaps not. Useful as these strategies may be in theory, they would undoubtedly come at a price. Only by finding it will we be in a position to evaluate the true measure of their success.

\section{ACKNOWLEDGMENTS}

This work was supported by National Institute of Health (NIH) grants R01-GM067247 and P50-GM076516

\section{REFERENCES}

\footnotetext{
Adachi-Yamada T, O’Connor MB. 2002. Morphogenetic apoptosis: a mechanism for correcting discontinuities in morphogen gradients. Developmental Biol 251: 74-90.

Akiyama T, Kamimura K, Firkus C, Takeo S, Shimmi O, Nakato H. 2008. Dally regulates Dpp morphogen
} 
gradient formation by stabilizing Dpp on the cell surface. Developmental Biol 313: 408-419.

Amonlirdviman K, Khare NA, Tree DR, Chen WS, Axelrod JD, Tomlin CJ. 2005. Mathematical modeling of planar cell polarity to understand domineering nonautonomy. Science 307: 423-426.

Ashe HL, Briscoe J. 2006. The interpretation of morphogen gradients. Development 133: 385-394.

Ben-Zvi D, Shilo BZ, Fainsod A, Barkai N. 2008. Scaling of the BMP activation gradient in Xenopus embryos. Nature 453: $1205-1211$.

Bergmann S, Sandler O, Sberro H, Shnider S, Schejter E, Shilo BZ, Barkai N. 2007. Pre-steady-state decoding of the Bicoid morphogen gradient. PLoS Biol 5: e46.

Bollenbach T, Kruse K, Pantazis P, Gonzalez-Gaitan M, Julicher F. 2005. Robust formation of morphogen gradients. Phys Rev Lett 94: 018103.

Bollenbach T, Pantazis P, Kicheva A, Bokel C, Gonzalez-Gaitan M, Julicher F. 2008. Precision of the Dpp gradient. Development 135: 1137-1146.

Cadigan KM, Fish MP, Rulifson EJ, Nusse R. 1998. Wingless repression of Drosophila frizzled 2 expression shapes the Wingless morphogen gradient in the wing. Cell 93: 767-777.

Carlson JM, Doyle J. 2002. Complexity and robustness. Proc Natnl Acad Sci 99: 2538-2545.

Crick FHC. 1970. Diffusion in embryogenesis. Nature 225: 420-422.

Eldar A, Dorfman R, Weiss D, Ashe H, Shilo BZ, Barkai N. 2002. Robustness of the BMP morphogen gradient in Drosophila embryonic patterning. Nature 419: 304-308.

Eldar A, Rosin D, Shilo BZ, Barkai N. 2003. Self-enhanced ligand degradation underlies robustness of morphogen gradients. Developmental Cell 5: 635-646.

Eldar A, Shilo BZ, Barkai N. 2004. Elucidating mechanisms underlying robustness of morphogen gradients. Curr Opin Genet Dev 14: 435-439.

Emberly E. 2008. Optimizing the readout of morphogen gradients. Phys Rev E Stat Nonlin Soft Matter Phys 77: 041903.

Feng XH, Derynck R. 1996. Ligand-independent activation of transforming growth factor (TGF) beta signaling pathways by heteromeric cytoplasmic domains of TGF-beta receptors. J Biol Chem 271: 13123-13129.

Fujise M, Takeo S, Kamimura K, Matsuo T, Aigaki T, Izumi S, Nakato H. 2003. Dally regulates Dpp morphogen gradient formation in the Drosophila wing. Development 130: $1515-1522$.

Giraldez AJ, Copley RR, Cohen SM. 2002. HSPG modification by the secreted enzyme Notum shapes the Wingless morphogen gradient. Developmental Cell 2: 667-676.

Gregor T, Tank DW, Wieschaus EF, Bialek W. 2007. Probing the limits to positional information. Cell 130: 153-164.

Han C, Yan D, Belenkaya TY, Lin X. 2005. Drosophila glypicans Dally and Dally-like shape the extracellular Wingless morphogen gradient in the wing disc. Development 132: 667-679.

Harris RA, Eichholtz TJ, Hiles ID, Page MJ, O’Hare MJ. 1999. New model of ErbB-2 over-expression in human mammary luminal epithelial cells. Int $J$ Cancer 80: 477-484.

Houchmandzadeh B, Wieschaus E, Leibler S. 2002. Establishment of developmental precision and proportions in the early Drosophila embryo. Nature 415: 798-802.

Khalsa O, Yoon JW, Torres-Schumann S, Wharton KA. 1998. TGF-beta/BMP superfamily members, Gbb-60A and Dpp, cooperate to provide pattern information and establish cell identity in the Drosophila wing. Development 125: 2723-2734.

Lander AD. 1999. Seeking the functions of cell surface heparan sulphate proteoglycans. In Cell Surface Proteoglycans in Signalling and Development (Human Frontiers Science Program Workshop VI) (ed. A.D. Lander, H. Nakato, S. Selleck, J. Turnbull, C. Coath), pp. 73-87. HFSP, Strasbourg.

Lauffenburger DA, Linderman JJ. 1993. Receptors. Models for binding, trafficking and signaling. Oxford University Press, New York.

Lecuit T, Cohen SM. 1998. Dpp receptor levels contribute to shaping the Dpp morphogen gradient in the Drosophila wing imaginal disc. Development 125: 4901-4907.

McHale P, Rappel WJ, Levine H. 2006. Embryonic pattern scaling achieved by oppositely directed morphogen gradients. Physical Biol 3: 107-120.

Morishita Y, Iwasa Y. 2008. Optimal placement of multiple morphogen sources. Phys Rev E Stat Nonlin Soft Matter Phys 77: 041909.

Moser M, Campbell G. 2005. Generating and interpreting the Brinker gradient in the Drosophila wing. Developmental Biol 286: 647-658.

Nellen D, Burke R, Struhl G, Basler K. 1996. Direct and longrange action of a DPP morphogen gradient. Cell 85: 357-368.

Neumann CJ, Cohen SM. 1997. Long-range action of Wingless organizes the dorsal-ventral axis of the Drosophila wing. Development 124: 871-880.

Reeves GT, Fraser SE. 2009. Biological systems from an engineer's point of view. PLoS Biol 7: e21.

Reeves GT, Muratov CB, Schupbach T, Shvartsman SY. 2006. Quantitative models of developmental pattern formation. Developmental Cell 11: 289-300.

Shimmi O, Umulis D, Othmer H, O’Connor MB. 2005. Facilitated transport of a Dpp/Scw heterodimer by Sog/Tsg leads to robust patterning of the Drosophila blastoderm embryo. Cell 120: 873-886.

Tabata T, Kornberg TB. 1994. Hedgehog is a signaling protein with a key role in patterning Drosophila imaginal discs. Cell 76: 89-102.

Tanimoto H, Itoh S, ten Dijke P, Tabata T. 2000. Hedgehog creates a gradient of DPP activity in Drosophila wing imaginal discs. Mol Cell 5: 59-71.

Tostevin F, ten Wolde PR, Howard M. 2007. Fundamental limits to position determination by concentration gradients. PLoS Comput Biol 3: e78.

White RJ, Nie Q, Lander AD, Schilling TF. 2007. Complex regulation of cyp26al creates a robust retinoic acid gradient in the zebrafish embryo. PLoS Biol 5: e304.

Zecca M, Basler K, Struhl G. 1996. Direct and long-range action of a wingless morphogen gradient. Cell 87: 833-844. 


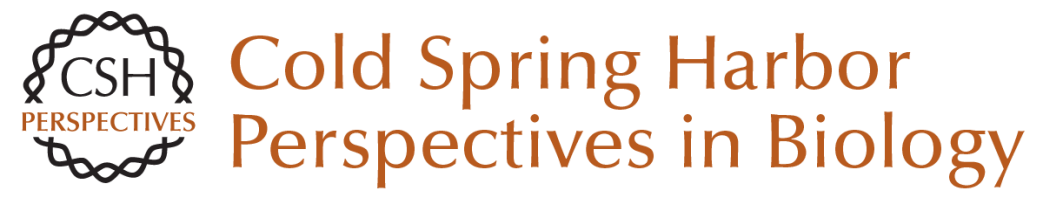

\section{The Measure of Success: Constraints, Objectives, and Tradeoffs in Morphogen-mediated Patterning}

Arthur D. Lander, Wing-Cheong Lo, Qing Nie and Frederic Y.M. Wan

Cold Spring Harb Perspect Biol 2009; doi: 10.1101/cshperspect.a002022

Subject Collection Generation and Interpretation of Morphogen Gradients

Regulation of Organ Growth by Morphogen

Gradients

Gerald Schwank and Konrad Basler

Signaling Gradients during Paraxial Mesoderm

Development

Alexander Aulehla and Olivier Pourquié

Morphogen Gradient Formation

Ortrud Wartlick, Anna Kicheva and Marcos

González-Gaitán

Nodal Morphogens

Alexander F. Schier

Gradients and the Specification of Planar Polarity in the Insect Cuticle

David Strutt

Vertebrate Limb Development: Moving from

Classical Morphogen Gradients to an Integrated

4-Dimensional Patterning System

Jean-Denis Bénazet and Rolf Zeller

Establishing and Interpreting Graded Sonic

Hedgehog Signaling during Vertebrate Neural

Tube Patterning: The Role of Negative Feedback

Vanessa Ribes and James Briscoe
Gradients in Planarian Regeneration and

Homeostasis

Teresa Adell, Francesc Cebrià and Emili Saló

Shaping Morphogen Gradients by Proteoglycans

Dong Yan and Xinhua Lin

Forming Patterns in Development without

Morphogen Gradients: Scattered Differentiation

and Sorting Out

Robert R. Kay and Christopher R.L. Thompson

Robust Generation and Decoding of Morphogen

Gradients

Naama Barkai and Ben-Zion Shilo

Models for the Generation and Interpretation of

Gradients

Hans Meinhardt

Graded Dorsal and Differential Gene Regulation in

the Drosophila Embryo

Gregory T. Reeves and Angelike Stathopoulos

Chemical Gradients and Chemotropism in Yeast Robert A. Arkowitz

For additional articles in this collection, see http://cshperspectives.cshlp.org/cgi/collection/

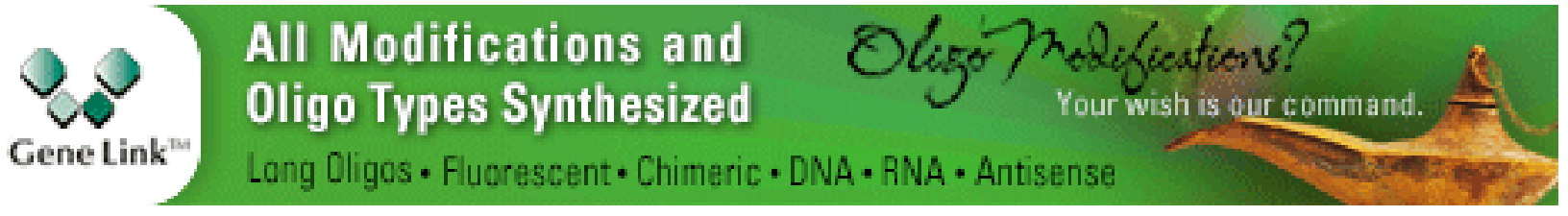

Copyright @ 2009 Cold Spring Harbor Laboratory Press; all rights reserved 
Systems Biology of the Self-regulating

Morphogenetic Gradient of the Xenopus Gastrula Jean-Louis Plouhinec and E. M. De Robertis
Gradients in the Brain: The Control of the Development of Form and Function in the Cerebral Cortex

Stephen N. Sansom and Frederick J. Livesey

For additional articles in this collection, see http://cshperspectives.cshlp.org/cgi/collection/

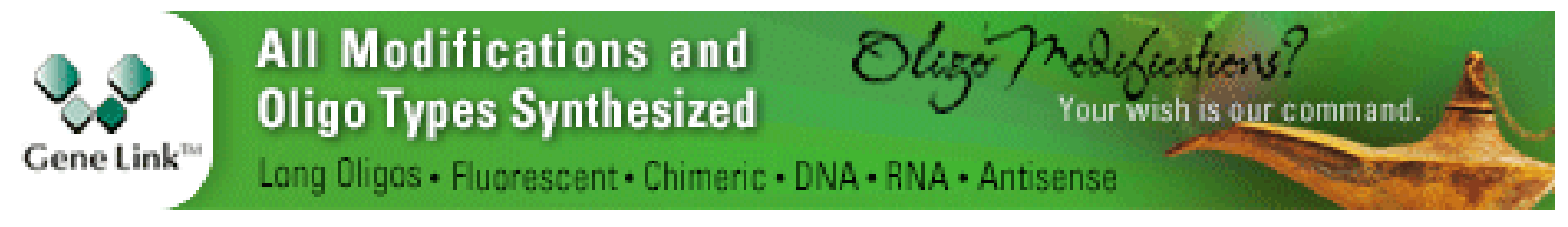

Copyright @ 2009 Cold Spring Harbor Laboratory Press; all rights reserved 